\title{
Riqueza de especies y abundancia de aves residentes y migratorias en parques urbanos de San José, Costa Rica
}

\author{
Aarón Fallas Solano \\ Escuela de Ciencias Exactas y Naturales, Universidad Estatal a Distancia (UNED), 2050 San José, Costa Rica; afallas@uned.ac.cr \\ Recibido 19-X-2017 • Corregido 14-XI-2017 • Aceptado 08-XII-2017
}

\begin{abstract}
Species diversity and abundance of resident and migratory birds in urban parks of San José, Costa Rica. With the exception of a few large cities, there is little knowledge about the urban birds of Latin America. I used linear transects in 15 urban parks in San José city between September and December, 2009 and found 40 species, mostly Tyrannidae $(n=10)$ and Parulidae $(n=9)$. There were 23 residents (mostly Tyrannidae), 15 northern migrants (mostly Parulidae) and one southern migrant. The most representative species were Contopus sp. and Setophaga petechia. Zenaida asiatica, Turdus grayi, and Quiscalus mexicanus were widespread, as was the pigeon, Columba livia, which could reach 122 individuals per park. Diversity and abundance were determined by park size, the complexity of vegetation and the proximity to vegetation fragments. The urban parks are being used as passing and permanent areas by the migratory birds and the biophysical characteristics of some parks favor Columba livia and Quiscalus mexicanus, generating competition, predation and displacement of another species, as well as infrastructure damage. These green areas could be used for bird watching and education.
\end{abstract}

Keywords: Urban park, green areas, urban ecology, urban biodiversity, resident birds, migratory birds.
RESUMEN: Con la excepción de unas pocas ciudades grandes, hay poco conocimiento sobre las aves urbanas de América Latina. Utilicé transectos lineales en 15 parques urbanos en la ciudad de San José entre setiembre y diciembre de 2009 y encontré 40 especies, la mayoría Tyrannidae $(n=10)$ y Parulidae $(n=9)$. Hubo 23 residentes (principalmente Tyrannidae), 15 migrantes del norte (en su mayoría Parulidae) y un migrante del sur. Las especies más representativas fueron Contopus sp. y Setophaga petechia. Zenaida asiatica, Turdus grayi y Quiscalus mexicanus estaban muy extendidos, al igual que la paloma Columba livia, que podía llegar a 122 individuos por parque. La diversidad y la abundancia fueron determinadas por el tamaño del parque, la complejidad de la vegetación y la proximidad a los fragmentos de vegetación. Los parques urbanos están siendo utilizados como áreas transitorias y permanentes por las aves migratorias y las características biofísicas de algunos parques favorecen a Columba livia y Quiscalus mexicanus, generando competencia, depredación y desplazamiento de otra especie, así como daños a la infraestructura. Estas áreas verdes podrían usarse para la observación y educación de aves.

Palabras clave: Parque urbano, áreas verdes, ecología urbana, biodiversidad urbana, aves residentes, aves migratorias.
Los estudios de avifauna en los sistemas urbanos en América Latina, se han concentrado en ciudades populosas de Suramérica (Mar de Plata, Buenos Aires, Sao Paulo, Santiago, Cali, Montevideo, La Paz, Arequipa) y México. En Argentina en la ciudad de Mar de Plata, se encontró que la riqueza, y abundancia de aves aumenta en las áreas suburbanas y está relacionada con la proporción de vegetación (Leveau \& Leveau, 2004). Así mismo, en Buenos Aires, un $20 \%$ de la totalidad de especies del país habitan en esta ciudad y sus alrededores. En Sao Paulo, Brasil se ha registrado la más alta riqueza de avifauna de las ciudades de América Latina con más de 140 especies en fragmentos de vegetación y parques. En La Paz, Bolivia, la composición del ensamble estaba influenciada de forma diferencial por los distintos niveles de urbanización y también por el gradiente altitudinal. Tanto en La Paz como en El Alto, la diversidad y riqueza de aves fueron bajas, porque existen muy pocas áreas verdes con superficie mayor a los $10.000 \mathrm{~m}^{2}$ y cobertura vegetal mayor al 50\% (Zavala \& Gismondi, 2000) En Chile, las comunidades de aves son dominadas por pocas especies muy abundantes (González, 2009). Además, se registró que los parques más grandes presentan un mayor número de especies que los de menor superficie (Urquiza \& Mella, 2002). En la ciudad de México, la composición y riqueza de especies cambian según las etapas de la urbanización, disminuyendo la diversidad al avanzar este proceso. Así mismo, los patrones de diversidad están asociados a características del hábitat (e.g., riqueza de especies arbóreas, cobertura y altura del estrato 
arbustivo, altura del estrato herbáceo) (González, 2009). Mientras que en América Central son escasas las investigaciones sobre avifauna urbana. Costa Rica, por ejemplo, presenta solo estudios de avifauna urbana en zonas aledañas a cursos de agua (Espinoza et al., 2002), estudios de especies invasoras (Ramírez et al. 2008) y en parques urbanos (Artavia \& Valle, 2013).

La importancia del estudio de la avifauna en parques urbanos reside en identificar la riqueza de especies de aves urbanas para determinar la importancia de los parques como hábitat (temporal o permanente) para estas especies. Además, de la importancia de que sean tomadas en cuenta en el desarrollo o implementación del diseño de infraestructura natural (parques o jardines construidos) para beneficio de la biodiversidad en las áreas urbanas. El objetivo de este estudio fue determinar la riqueza y abundancia de aves residentes y migratorias en parques urbanos del Cantón Central de San José.

\section{MATERIALES Y MÉTODOS}

El trabajo de campo se llevó a cabo entre setiembre y diciembre del 2009, en 15 parques urbanos en la ciudad de San José, Costa Rica pertenecientes a cuatro distritos del Cantón Central de San José: El Carmen, Catedral, Hospital y Merced. La topografía del cantón es regular con una ligera inclinación de este a oeste, presenta elevaciones entre 950 y 11 190msnm (Barrantes, 1964). Específicamente para la provincia de San José presenta una temperatura promedio de $20,3^{\circ} \mathrm{C}$ y una precipitación anual que oscila entre 1500 - 2000 mm (Murillo \& León, 2007). Los parques urbanos estudiados fueron: Parque Morazán, Jardín de Paz, Parque España, Parque Nacional, Plaza de la Democracia, Parque de las Garantías Sociales, Plaza de las Artes, Plaza González Víquez, Parque Cañas, Parque Dolorosa, Parque Central, Parque Ministerio de Salud, Parque Braulio Carrillo, Bulevar del Correo y Parque Central de Barrio México (Fig. 1).

Los parques urbanos se seleccionaron aleatoriamente mediante el establecimiento de una división imaginaria del cantón central de San José en cuatro cuadrantes según los puntos cardinales. Se utilizó como punto de referencia calle 0 y avenida 0 definiendo cada cuadrante según los puntos cardinales. Todos los parques y áreas verdes que se encuentren en esa área, fueron tomados como sitios de estudio siempre y cuando presentaran las siguientes variables: 1) área superior $1700 \mathrm{~m}^{2}, 2$ ) parques urbanos ubicados en un radio de $1 \mathrm{Km}$ a partir de calle 0 avenida 0 y 3) administrados por la Municipalidad de San

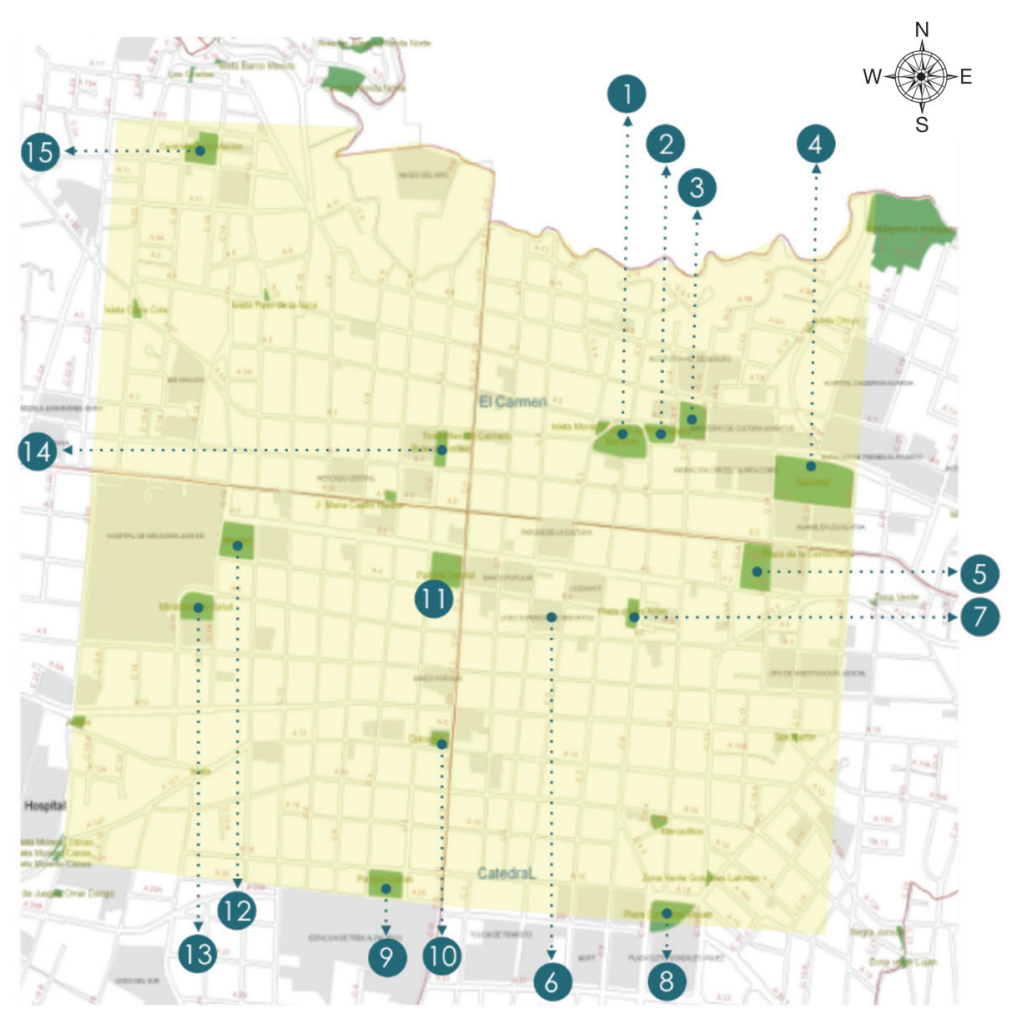

Simbología:

1. Parque Morazán

2. Jardín de Paz

3. Parque España

4. Parque Nacional

5. Plaza de la Democracia

6. Parque Garantias Sociales (CCSS)

7. Plaza de las Artes

8. Plaza González Víquez

9. Parque Cañas

10. Parque Dolorosa

11. Parque Central

12. Parque Braulio Carrillo

13. Parque Ministerio de Salud

14. Bulevar del Correo

15. Parque Central de Barrio México

Fig. 1. Distribución de los parques urbanos muestreados del Cantón Central de San José, Costa Rica. Adaptado de Fallas (2016). 
José, ya que este gobierno local está realizando acciones en la temática ambiental. Los parques urbanos en estudio (Cuadro 1 en Apéndice Digital) presentan diferentes características entre sí, en cuanto a superficie, coordenadas y cuadrante de ubicación.

Determinación de la riqueza y abundancia de aves: La riqueza y abundancia de las aves se determinó mediante el método de transecto en franjas (Ralph et al., 1996). Se realizaron tres muestreos por parque (entre las 06:00 y las 10:00 horas) haciendo recorridos (sin paradas) dentro de los senderos internos de cada parque y las aceras perimetrales. Durante el recorrido se anotó la cantidad de individuos por especie y la característica del sitio donde se encontraba (suelo o pavimento, estrato vegetal, infraestructura). Los muestreos se realizaron con una frecuencia de dos veces por semana y se distribuyeron de la siguiente manera: la secuencia de muestreo fue de forma ordenada del cuadrante 1 al 4 . El esfuerzo de muestreo se estandarizó por área (metros cuadrados).

Se determinó el número de especies (validando nombre de las especies con la lista oficial de aves de Costa Rica) para obtener la riqueza y la abundancia fue determinada por el número de individuos por especies por parque. Para el análisis de la riqueza y abundancia de especies de aves, se realizó una comparación entre parques, utilizando la prueba no paramétrica, de Kruskal-Wallis (Sokal \& Rolft, 1995); la cual fue validada previamente mediante pruebas de homogeneidad (Test de Levene y Bartlett) y de normalidad (Test de Shapiro-Wilk) donde en ambas $P<0,05$, esto asegura la necesidad de aplicar una prueba no paramétrica como Kruskal-Wallis. Dichas pruebas fueron calculadas mediante el paquete estadístico R. Además, se aplicó el índice de diversidad de Simpson el cual permitió determinar la especie más dominante. Así como, el índice de similitud de Morisita-Horn que permitió cuantificar semejanza entre parques; ambas mediciones mediante el paquete estadístico Past3 (Hammer, Harper \& Ryan, 2001).

Se elaboró una curva de rarefacción basada en muestreos para determinar qué tan cerca fue posible llegar del número esperado de especies para ese sitio. Esta curva, la cual es una expectativa estadística de la curva de acumulación de especies, calcula el número esperado de especies cuando $\mathrm{n}$ número de muestreos son escogidos al azar (Gotelli \& Colwell, 2010). La misma se eleva rápidamente a medida que se va acumulando el número de especies debido al incremento en el número de muestreos (i.e. área cubierta), pero disminuye conforme se añaden especies raras hasta llegar a formar una asíntota que indica que todas las especies para el área de muestreo han sido registradas (Ugland et al., 2003). Para elaborar este análisis se utilizaron todas las especies detectadas durante y fuera de los conteos. Los intervalos de confianza de la curva fueron calculados al 95\%. Además, se realizó una estimación de la riqueza de especies utilizando el estimador no-paramétrico Chao2 (Chao2, 1987) incluyendo las especies vistas. El estimador Chao2, que ha demostrado ser uno de los más útiles en varias comunidades bióticas (Walther \& Morand, 1998), incluyendo aves (Chazdon et al., 1998; Herzog et al., 2002), estima la riqueza total incluyendo aquellas especies no presentes en los muestreos; asimismo, es menos sensible a la heterogeneidad en la distribución de las especies y a la variabilidad en la probabilidad de encontrarlas (Hortal et al., 2006). Tanto la curva de rarefacción como el estimador de la riqueza Chao2 fueron calculados utilizando el programa EstimateS v.9 (Colwell, 2013). Se realizaron 100 corridas aleatorizadas y se extrapoló a un total de 80 muestreos.

\section{RESULTADOS}

Riqueza de especies de aves: Se determinó una riqueza total de 40 especies de aves (oscilando entre cuatro y 24 especies según el parque), distribuidas en seis ordenes, 15 familias. El orden con mayor representatividad de familias y especies fue Passeriformes con 10 familias de las cuales Tyrannidae $(S=10)$ y Parulidae $(S=9)$ fueron las familias con mayor cantidad de representantes. Las especies que registraron mayor cantidad de individuos para este orden fueron Quiscalus mexicanus $(\mathrm{S}=274)$ y Thraupis episcopis $(\mathrm{S}=72)$. El orden Columbiformes fue el siguiente en cuanto a cantidad de representantes, registrándose cuatro especies de palomas, y Columba livia fue la especie más abundante en toda el área de estudio, con 1726 individuos registrados (Cuadro 2 en Apéndice Digital).

El 57\% corresponden a especies residentes (R), el 37\% a migratorias norte (MN), mientras que $3 \%$ corresponde a especies residente-migratorias norte $(\mathrm{R}, \mathrm{MN})$, así como residente-migratorio sur (R, MS) (3\%) (Fig. 2). Sobresale

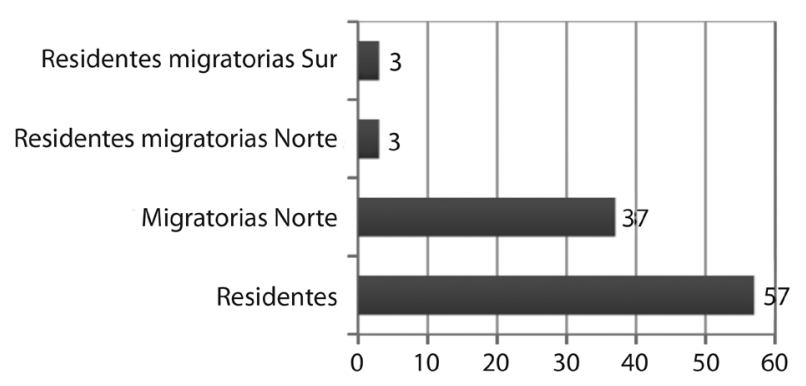

Fig. 2. Valores porcentuales de avifauna observada en 15 parques urbanos el Cantón Central de San José en el Periodo setiembre-diciembre 2009. 
Parulidae como la familia con mayor presencia de especies migratorias (9 especies), mientras que Tyrannidae como la familia con mayor cantidad de especies residentes (9 especies).

El cuadrante 1 fue el que presentó mayor riqueza de aves, registrándose un total de 31 especies, seguido del cuadrante 3 con 23 y el cuadrante 4 con 21 especies, mientras que el cuadrante 2 presentó menor riqueza, contabilizándose 18 especies (Fig. 3). Es importante tomar en cuenta que el cuadrante 4 solamente incluye dos sitios de muestreo, mientras que los otros tienen cuatro y cinco sitios muestreados y el cuadrante 1 incluye parques con mayor cobertura vegetal y están más cerca unos de otros, prácticamente interconectados entre si y ubicados relativamente cerca a otras masas vegetales importantes como lo es el Parque Zoológico Simón Bolívar y el río Torres.

El Parque Nacional fue el sitio en el que se registró mayor riqueza de especies, con un total de 24 , donde las especies más frecuentes de observar fueron Quiscalus mexicanus (44) y Columba livia (29), Turdus grayi (11). En segundo lugar, se encuentra el Parque Morazán donde se observaron 23 especies, siendo C. livia y $Q$. mexicanus las especies de mayor observación en el sitio, mientras que en el Parque España se registraron 21 especies de aves en este caso las especies con mayor representación fueron T. grayi y T. episcopus. Por otro lado, el sitio con menor riqueza corresponde a La Plaza de las Artes, el cual presentó una riqueza de cuatro especies, además en este sitio se registraron pocos individuos por especie, incluso para C. livia (Cuadro 2 en Apéndice Digital).
Con respecto al estatus, es importante señalar que en todos los cuadrantes predominaron las especies residentes. En el cuadrante 1 se registraron 22 especies residentes y 10 especies migratorias. En el cuadrante 2 se contabilizaron 11 especies en estatus residente y ocho en estatus de migratoria. Por su lado, el cuadrante 3 se registró 14 especies residente y solamente siete especies migratorias. Tanto en el cuadrante 1, como el 2 y el 3 se encuentra una especie residente con parte de la población migratoria latitudinal norte (Contopus virens). Por su parte, en el cuadrante 4 se registraron 14 especies residentes y 9 especies migratorio (Fig. 3). Es importante mencionar que solo en este cuadrante se registró Pygochelidon cyanoleuca (Cuadro 2 en Apéndice Digital).

El Parque Nacional fue el sitio en el que se registró mayor riqueza de especies residentes, observándose un promedio de 18,5 migratorias del norte y una residente con parte de la población migratoria norte. Por otro lado, el sitio con menor riqueza corresponde a La Plaza de las Artes, el cual presentó una riqueza de cuatro especies de aves, Columba livia y Quiscalus mexicanus (R) y Vermivora chrysoptera y Setophaga petechia (MN). Es importante mencionar que en 9 parques se identificaron especies con estatus de residente-migratorio norte, mientras que solo en uno de ellos se identifica una especie con estatus residente-migratorio sur (Pygochelidon cyanoleuca) (Fig. 4).

Existe una alta presencia de especies de aves principalmente residentes en el Parque Nacional y el Parque Morazán (cuadrante 1). Por el contrario, en los tres cuadrantes restantes se observó una dispersión y aislamiento

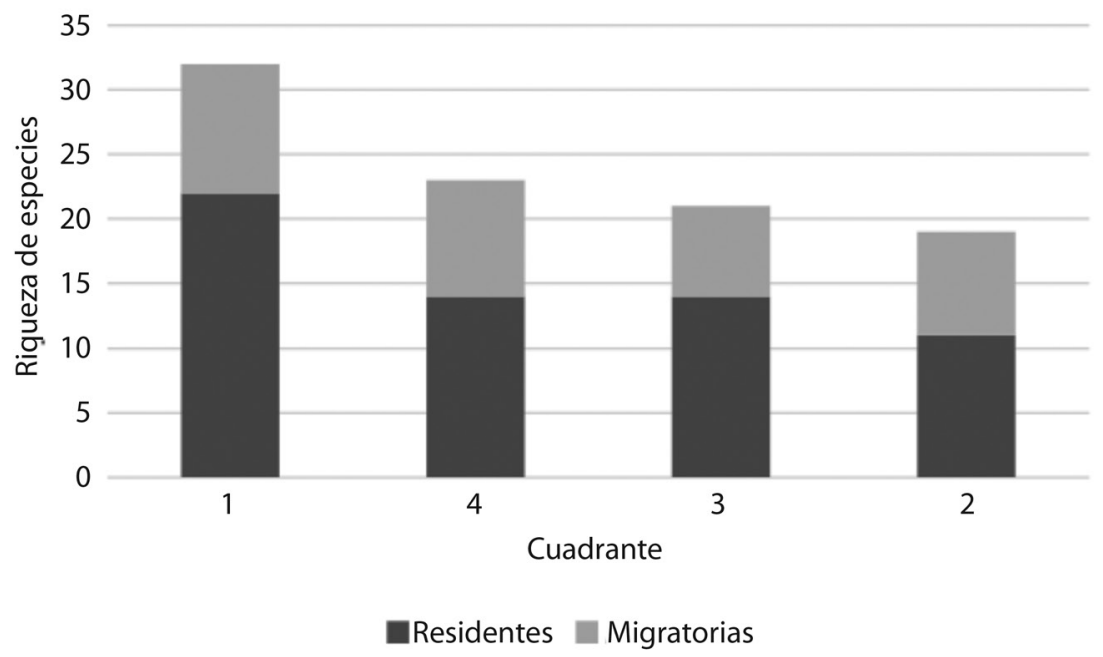

Fig. 3. Riqueza y estatus paras aves registradas en cada cuadrante analizado en el Cantón Central de San José en el período setiembre-diciembre 2009. 


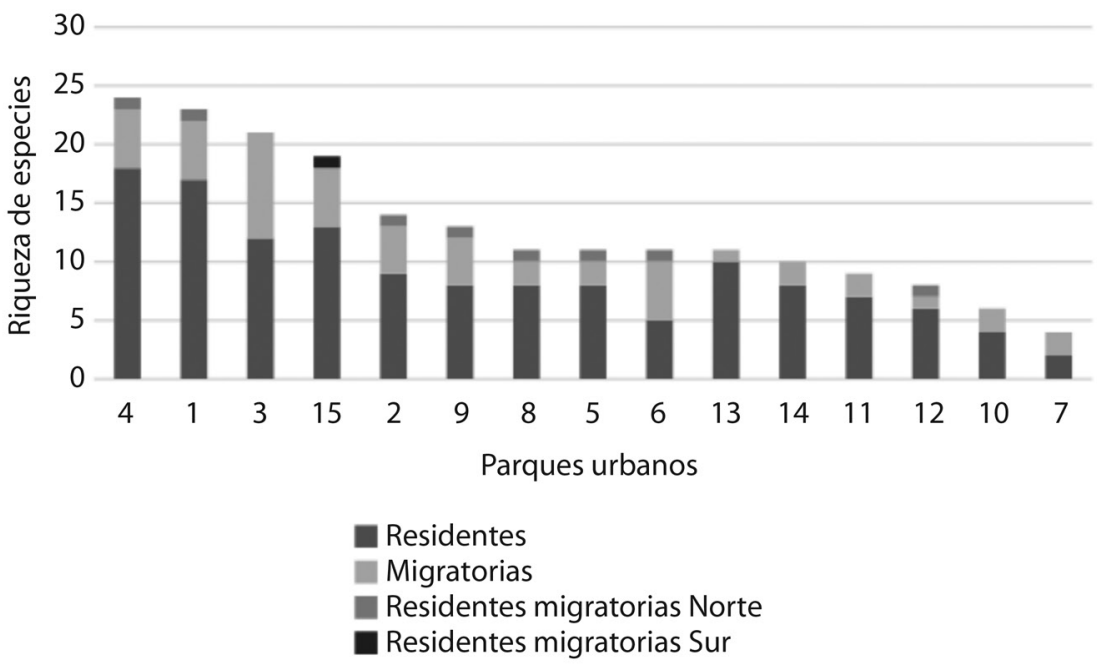

Fig. 4. Riqueza y estatus paras aves registradas en cada parque urbano analizado en el Cantón Central de San José en el periodo setiembre-diciembre 2009.

entre las zonas verdes o parques en estudio con ausencia de conectividad vegetal entre ellas (fragmentación), asimismo, no se muestran indicios de conectividad con fragmentos ni espacios verdes cercanos de importancia (ajenos al área de estudio).

A partir del índice de diversidad de Simpson se determina la dominancia de especies, este indica que los valores más cercanos a 1 son los sitios con mayor diversidad de aves (dominancia alta), los cuales corresponden a los parques 2-3-4-8-9 y 15, en donde el parque 3 (Parque España) presenta la mayor diversidad de especies de aves. Contrastando con lo observado en campo los parques 1-3-4-15 son los que presentan mayor riqueza de especies de aves, reforzando en 3 parques (3-4-15) lo generado por el índice de diversidad de Simpson (Cuadro 3 en Apéndice Digital).

Los parques 1-6-11-12 y 14 son los que presentan menor diversidad (dominancia menor a $>0.5$ ), siendo el parque 14 (Bulevar del Correo) el que presenta menor diversidad de aves, en estos sitios se da una mayor abundancia y dominancia de Columba livia, ya que representan un hábitat idóneo para la presencia de esta especie. Contrastando con lo observado en campo los parques 7-10 son los que presentan menor riqueza de especies de aves, lo cual no concuerda con los datos generados por el índice de diversidad de Simpson (Cuadro 3 en Apéndice Digital).

Los datos de riqueza de especies de aves generadas a partir del trabajo de campo, se acercan por mucho en los cuadrantes 1-2-3 a la estimación de la riqueza generada a partir del estadístico Chao 2. Particularmente el cuadrante 4 es el que presenta una discordancia entre el número de especies observadas en campo y lo estimado por el estadístico Chao2 (Cuadro 4 en Apéndice Digital).

La riqueza de especies de aves extrapolada, muestra un incremento positivo a un nivel de confianza del $95 \%$. A partir de la curva de acumulación de especies, se esperaría encontrar una riqueza de por lo menos 122 especies al aumentar ya sea el número de muestreos o el número de sitios (como mínimo 45 muestreos en el tiempo). Esto concuerda con lo que indica el dato de Chao2 en el cual se interpreta que es necesario generar más muestreos que permitan estabilizar la curva ya que el número es más alto del observado (Fig. 5).

Mediante el índice de similitud de Morisita se comparó la composición de especies de aves por parque y se determinó que existen 2 grandes grupos con 2 subgrupos cada uno. Un primer grupo integrado por los parques 5-7-10-6-1-11-12-14 con una similitud aproximada al $90 \%$ y del cual se subdivide en 2 subgrupos. Así mismo el segundo grupo lo integran los parques 2-3-4-8-9-1315 con una similitud del $45 \%$ entre la riqueza y abundancia de especies.

El primer subgrupo (observado a partir del grupo con 90\% de similitud) está integrado por los parques 5-7-10 con una similitud aproximada al 97\% (Fig. 6). Este grupo se caracteriza por ser parques con áreas relativamente pequeñas respecto a todos los sitios de estudio $\left(8382 \mathrm{~m}^{2}\right.$ $-1983 \mathrm{~m}^{2}$ y $1786 \mathrm{~m}^{2}$ respectivamente), con un porcentaje promedio de áreas verdes que no supera el $20 \%$, por consiguiente un $80 \%$ de superficies pavimentadas. Además, presentan bajo porcentaje tanto de abundancia, como 


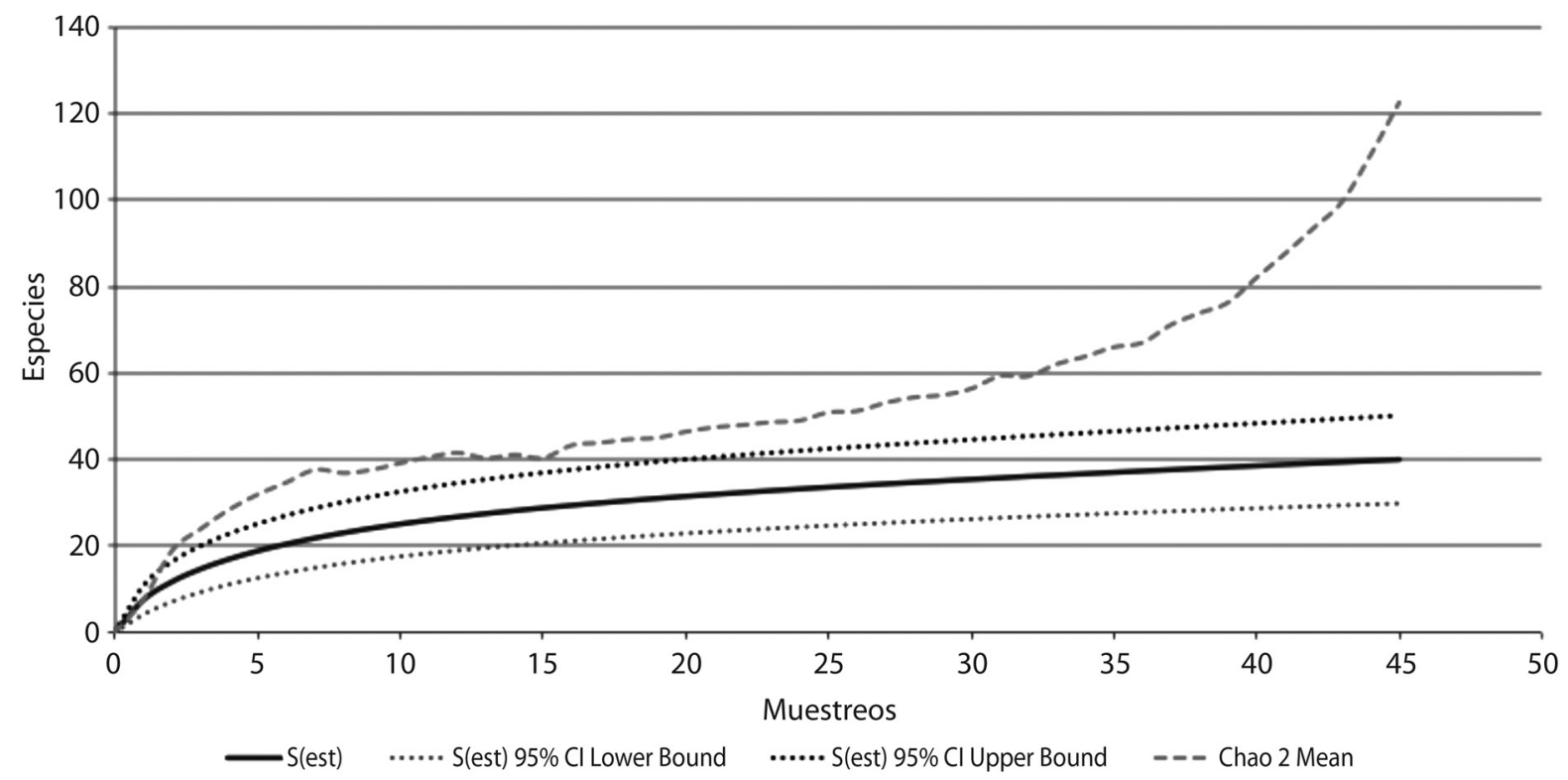

Fig. 5. Curva de acumulación de especies de aves y la estimación de la riqueza en los sitios de estudio dentro del Cantón Central de San José, en el periodo setiembre-diciembre 2009.

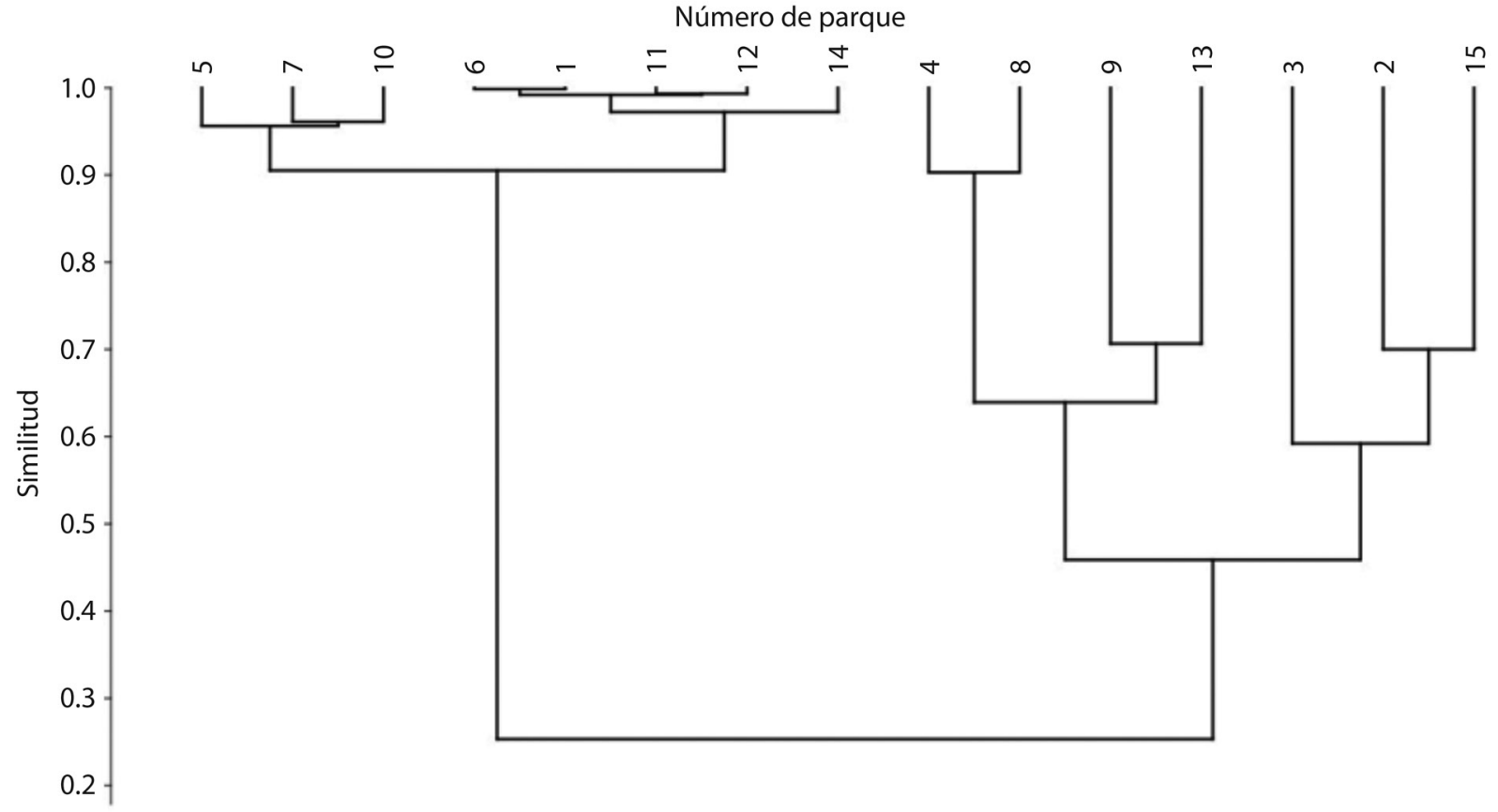

Fig. 6. Índice de similitud de Morisita de especies de aves en los parques, Cantón Central de San José.

de riqueza (rango de 4-11 especies), con respecto a los otros grupos donde sobresale Columba livia como especie más abundante (35 individuos promedio). Se considera que la similitud en este grupo se genere debido a las marcadas diferencias en bajos porcentajes de área verde, alto porcentaje de áreas pavimentadas (característica que comparte con el segundo grupo y bajos porcentajes tanto de riqueza como abundancia.

El segundo subgrupo (observado a partir del grupo con $90 \%$ de similitud) está compuesto por los parques 6-1-11-12-14 con una similitud aproximada al 95\%. (Fig.6). Este grupo se caracteriza por ser parques 
con áreas que superan en su gran mayoría los $6000 \mathrm{~m}^{2}$ $\left(5966 \mathrm{~m}^{2}, 9378 \mathrm{~m}^{2}, 6312 \mathrm{~m}^{2}, 6917 \mathrm{~m}^{2}\right.$ y $2507 \mathrm{~m}^{2}$ respectivamente), con un porcentaje promedio de áreas verdes bajo, en tres de los sitios no supera el 18\%, mientras que los dos restantes no superan el $48 \%$, predominan las áreas pavimentadas dos de los sitios con porcentajes por encima de $50 \%$ y los tres restantes superan el $80 \%$. Este grupo presenta un alto porcentaje de abundancia donde sobresale Columba livia como especie más abundante (por encima de 300 individuos promedio) y una riqueza promedio que ronda las 10 especies (rango general de 8-11 especies), sin embargo, sobresale el parque nacional como caso aislado, en donde la riqueza alcanza las 23 especies (segundo sitio con mayor riqueza según datos generados en el estudio). Se considera que la similitud en este grupo se genere debido a las marcadas diferencias en bajos porcentajes de área verde, alto porcentaje de áreas pavimentadas (característica que comparte con el primer grupo), así como un alto porcentaje de abundancia y una riqueza intermedia según promedio de todos los parques estudiados.

Los 2 subgrupos observados a partir del grupo con $45 \%$ de similitud, lo integran parques con menor similitud $(60 \%$ y $62 \%)$ entre la riqueza y abundancia de especies (parques 2-3-4-8-9-13-15) (Fig. 6), indicando que son sitios donde existe mayor diversidad de especies (15 en promedio por parque con un rango que oscila entre 11 y 24). Presentan un rango de abundancia que va de 11 a 49 individuos. En estos sitios no se evidencia abundancia de Columba livia y las especies observados corresponden mayormente a especies migratorias. Se considera que la relación de variables que genere que se agrupen es la relación es el equilibrio porcentual entre áreas verdes y áreas pavimentadas, ya que en cinco de los siete sitios dicha relación guarda un equilibrio porcentual que no supera el $10 \%$ de diferencia entre área verde y área pavimentada. Mientras que el comportamiento de estas variables es contrario en uno y otro sitio (uno presenta superioridad porcentual de área verde sobre área pavimentada (81\%-19\% respectivamente), mientras que el otro presenta mayor porcentaje de área verde sobre área pavimentada (30\%-70\% respectivamente). También sobresale la relación entre abundancia (inferior a 49 individuos) y riqueza (alto porcentaje). Encontrándose dentro de este grupo los sitios más diversos (en excepción el Parque Nacional, el cual por las diferencias entre las variables no se encuentra en este).

Estos datos permiten identificar y apoyar lo observado, e indicar cuan importantes son las variables contempladas en lo que respecta a los requerimientos de hábitat para las especies que algunos parques urbanos del cantón Central de San José contemplan, variables que favorecen (según corresponda) la presencia de especies nativas, migratorias o exóticas.

Abundancia de especies de aves: La abundancia promedio de individuos por parques fue de 173,06, (DE= $177,6)$ con un ámbito de oscilación entre 9 y 647, indicando que la mayoría de los promedios de individuos por parque son similares al promedio, sin embargo, el rango de oscilación de datos esta entre 9 y 647 individuos, siendo los picos la Plaza de las Artes y el Bulevar del Correo respectivamente, evidenciando la disparidad en cuanto a la abundancia de aves.

La abundancia de aves en los sitios de estudio mostró una diferencia biológica evidente, sin embargo, no existe una diferencia estadística $\left(\mathrm{Chi}^{2}=13,9\right.$, nivel de $\mathrm{Chi}^{2}$ corregido: 14 significancia: 0,4497$)$ que respalden las diferencias biológicas identificadas.

Se contabilizó un total de 2596 individuos en el área de estudio, en donde la familia con mayor abundancia fue Columbidae, representando el $70 \%$ de los datos, donde Columba livia es la especie más abundancia para esta familia (1726 individuos registrados), seguida por la familia Icteridae donde las especies Quiscalus mexicanus $(\mathrm{S}=274)$ y Thraupis episcopis $(S=72)$ tuvieron mayor frecuencia. El cuadrante 3 es el que presentó la mayor abundancia de aves (teniendo como especies más abundantes a C. livia y $Q$. mexicanus), mientras que el cuadrante 2 es el que presentó menor abundancia total de especies. La Plaza de las Artes, es el sitio donde se registraron la menor cantidad de individuos por especie, incluso para C. livia (Fig. 7).

El porcentaje de abundancia de especies de aves en tres de los cuatro cuadrantes (uno, tres y cuatro), fue similar (Cuadrante 1: 24\%, cuadrante 3: 30\% y Cuadrante 4: $29 \%$ respectivamente). Sin embargo, esta diferencia se aprecia la cantidad de especies observadas por cuadrante (Cuadrante $1=31$, cuadrante $2=18$, cuadrante $3=23$ y cuadrante 4=21) (Cuadro 5 en Apéndice Digital). El cuadrante 3 es el que presentó la mayor abundancia de aves (teniendo como especies más abundantes a $C$. livia y $Q$. mexicanus). Mientras que el cuadrante 2 es el que presentó menor abundancia total de especies, donde la participación de estas dos especies es similar al resto de los cuadrantes.

Abundancia por parque: Se identificaron 5 sitios con una abundancia mayor al promedio (295 individuos), sobresaliendo el Boulevard del Correo (1321 individuos), el parque Morazán (629 individuos), el parque Central (591 individuos), el parque Braulio Carrillo 


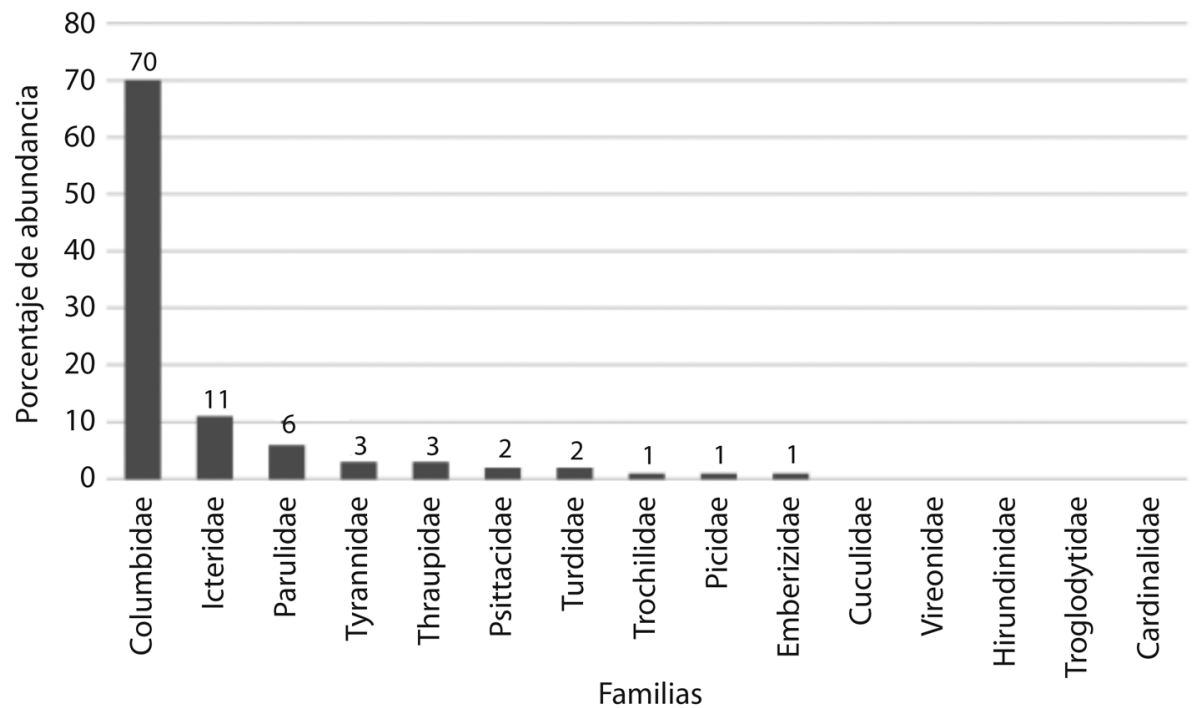

Fig. 7. Promedio de individuos por familia observado para cada parque en el cantón Central de San José en el periodo setiembre-diciembre 2009.

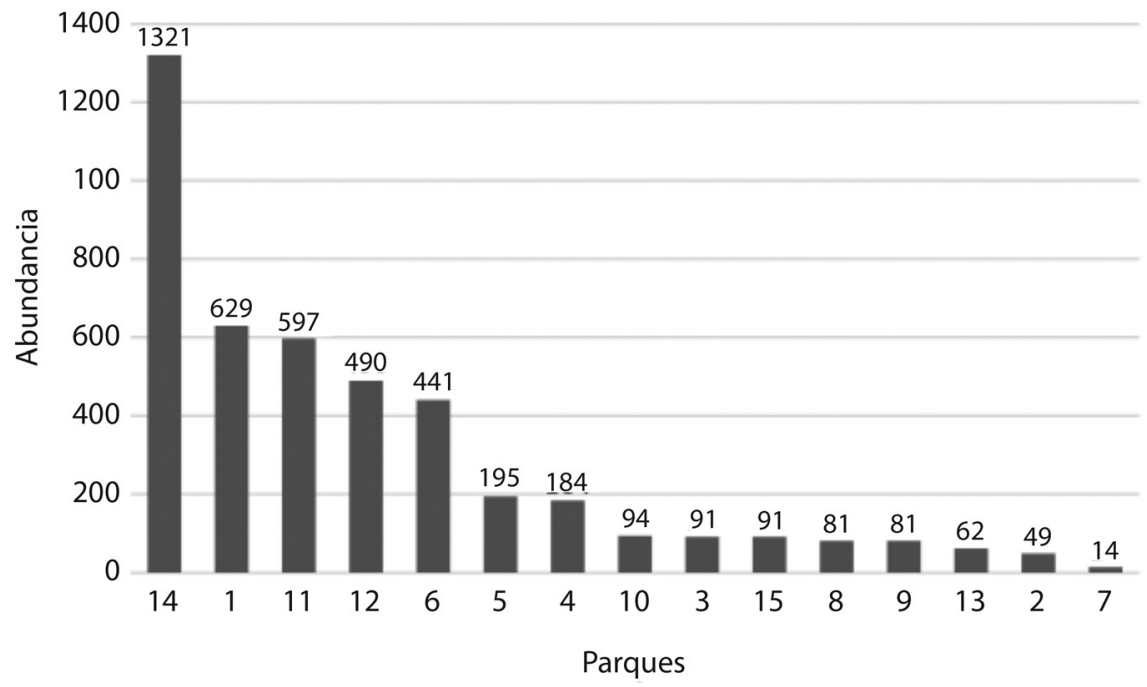

Fig. 8. Abundancia de especies de aves en los sitios de estudio dentro del Cantón Central de San José, en el periodo setiembre-diciembre 2009.

(Merced) (490 individuos) y las Garantías Sociales (441 individuos) (Fig. 8).

Es importante mencionar que en el estudio no se contempló (por razones de metodología, sitio no administrado por la Municipalidad de San José) la Plaza de la Cultura en el casco central, sitio que se convierte, sin lugar a duda, en el espacio público a nivel de San José con mayor abundancia de Columba livia, posiblemente debido a los factores antes mencionados.
Relación Riqueza-abundancia: El promedio de la riqueza y la abundancia de todos los parques (Fig. 9), se ven alterados por 4 parques (Plaza de las Artes, Parque de la Dolorosa, Parque Central y Parque Braulio Carrillo (Merced) los cuales condicionan los datos reales de riqueza y abundancia para los parques urbanos de San José al presentar una riqueza baja. Se observa una relación equitativa directa entre riqueza y abundancia en 8 parques, la cual se evidencia al comparar los parques 


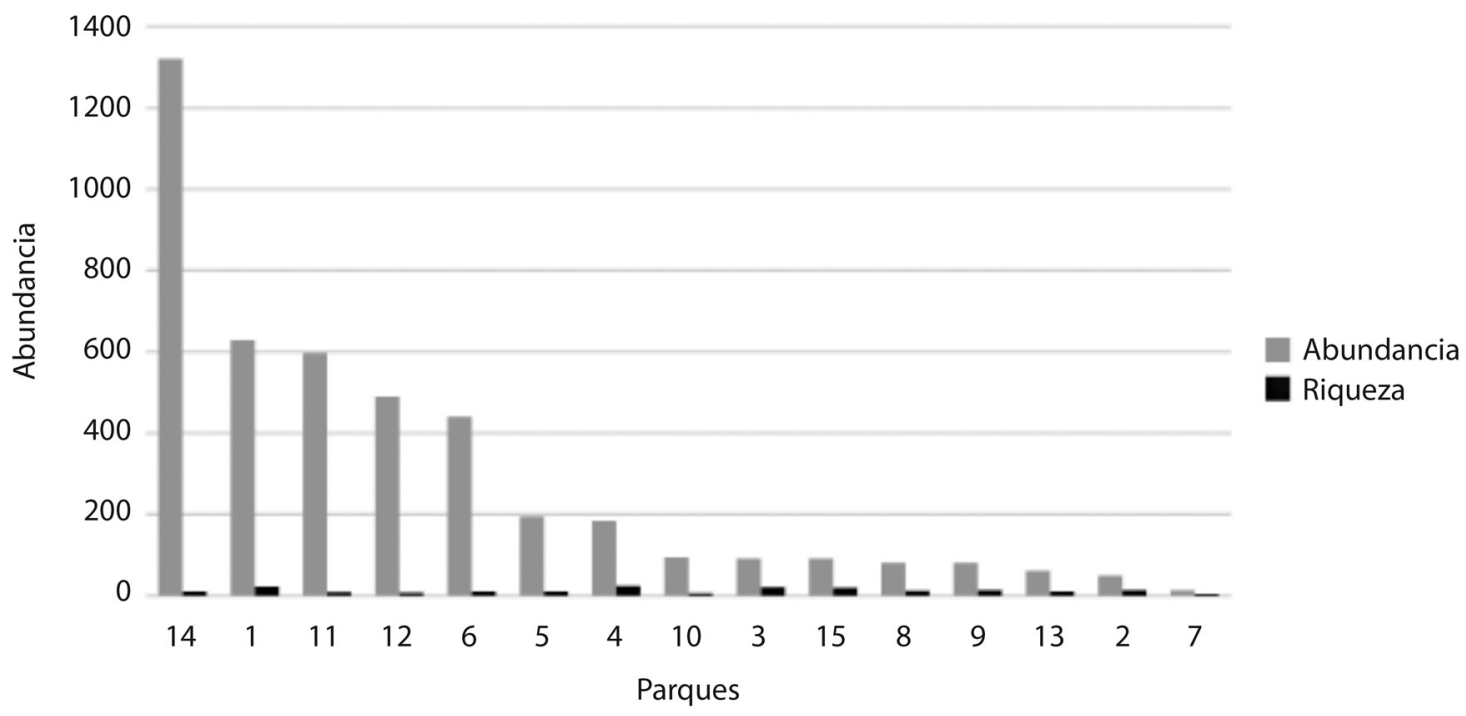

Fig. 9. Abundancia y riqueza de especies de aves en los sitios de estudio dentro del Cantón Central de San José, en el periodo setiembre-diciembre 2009.

(2-3-4-7-8-9-13-15 (Fig. 9) solo 2 de los parques (3-4) presentan estructuras vegetales complejas (características favorables para especies migratorias) que podrían determinar la riqueza y abundancia de aves, mientras que los restantes parques presentan árboles relativamente altos (aquellos que superan los $5 \mathrm{~m}$ de altura como cipreses, eucaliptos) que brindan recursos alimenticios a especies insectívoras migratorias.

Se encontró en 10 sitios una elevada riqueza (2-3-45-7-8-9-10-13-15) con abundancia relativamente baja, estos sitios corresponden a lugares que presentan características mencionadas que favorecen la riqueza de aves (tamaño del parque, la edad (habitualmente relacionada positivamente con la complejidad en la estructura de vegetación del parque) y por el aislamiento respecto del hábitat fuente) (Fig. 9).

\section{DISCUSIÓN}

El orden con mayor representatividad de familias y especies corresponden a Passeriformes con Tyrannidae y Parulidae; siendo una condición esperada pues son las familias con mayor cantidad de especies registradas en el país. De las especies encontradas las más abundantes son generalistas, como lo son Quiscalus mexicanus (Omnívoro), Zonotrichia capensis (granívoro, insectívoro), Thraupis episcopus (frugívoro-insectívoro) y Columba livia (granívoro-omnívoro), también se registraron especies de aves que requieren de hábitat menos alterados con concentraciones vegetales mayores, tales como Conopias albovittatus (no es la distribución habitual), Coccyzus americanus, Vermivora chrysoptera, Parkesia noveboracensis, entre otras; indicando con esto la importancia que tienen los parque urbanos como hábitat capaces de sustentar a especies de fauna con mayores requerimientos de hábitat en un medio altamente deteriorado.

La alta presencia de aves residentes en el Parque Nacional y el Parque Morazán puede deberse a la cercanía de los 4 parques contemplados bajo este cuadrante (los cuales están inmersos en menos de $500 \mathrm{~m}$ lineales), la presencia de masas vegetales (franja boscosa ribereña del río Torres), la poca presencia de superficies duras (áreas obra gris-pavimentos) y la conectividad de los mismos mediante un corredor paisajístico arbóreo denominado el paso de las Damas facilitando la obtención de agua, alimento y sitios de anidación por parte de las aves (necesidades básicas de las aves), generando un gran espacio, que puede ser identificado como un hábitat bloque. Esto también fue observado por Fernández-Juricic y Jokimäki (2001) quienes indican que las calles arbolados, parecen favorecer la conectividad general de los parques minimizando, el efecto del aislamiento (De la Hera et al., 2009). Además, como lo indica De la Hera et al. (2009), la riqueza de especies en los parques urbanos se explica en gran medida gracias al área total de los parques y al porcentaje de la cobertura arbórea, así como todas las áreas aledañas que permiten reducir el efecto de fragmentación en entornos urbanos. 
En cuadrantes donde se observó dispersión y/o aislamiento entre las zonas verdes con ausencia de conectividad vegetal entre ellas (fragmentación), se considera que la riqueza de especies de aves puede verse desfavorecida. De la Herra et al. (2009) citando a Soulé et al. (1988); Fernández-Juricic (2001) indican que la riqueza de especies de aves en los parques urbanos puede verse afectada por el tamaño del parque, la edad (habitualmente relacionada positivamente con la complejidad en la estructura de vegetación del parque) y por el aislamiento respecto del hábitat fuente.

Los datos de riqueza de especies de aves generadas a partir del trabajo de campo, se acercan por mucho en los cuadrantes 1-2-3 a la estimación de la riqueza generada a partir del estadístico Chao 2 (Cuadro 4), no así en el cuadrante 4. Las especies constantes (especies vistas en todos o casi todos los muestreos) pueden estar generando que el dato estadístico sea muy similar al dato observado en los cuadrantes 1-2-3. La pequeña diferencia generada en todos los cuadrantes puede deberse a la cantidad de especies esporádicas (vistas una o dos veces) principalmente se observan como especies esporádicas la Vermivora chrysoptera y Wilsonia canadensis (Cardellina canadensis), ambas especies migratorias esto para el cuadrante 2. Mientras que para el cuadrante 1 las especies esporádicas identificadas son: Empidonax spp (Migratorio), Elaenia flavogaster (Residente), Myiarchus spp (muchas posibilidades de ser residente, ya que de seis especies una es migratoria), Troglodytes aedon (Residente), Oporornis philadelphia (especie sin comprobante) y Piranga rubra (Migratorio). Y para el cuadrante 3 las especies esporádicas son: Brotogeris jugularis (Residente), Empidonax spp (Migratorio), Tyrannus melancholicus (Residente), Zonotrichia capensis (Residente) y Icterus galbula (Migratorio). La discordancia presente en el cuadrante 4 igualmente puede deberse a las especies esporádicas ya que la lista es mayor para este cuadrante. Para este cuadrante fueron vistas una o dos veces: Brotogeris jugularis (Residente), Coccyzus americanus (Migratorio), Melanerpes hoffmannii (Residente), Elaenia $s p$ (Residente), Pitangus sulphuratus (Residente), Vireo flavifrons (Migratorio), Catharus ustulatus (Migratorio), Dendroica pensylvanica (Setophaga petechia) (ResidenteMigratoria), Thraupis palmarum (Residente), Icterus galbula (Migratoria). La mayoría de estos corresponden a una bandada mixta observada en un único muestreo.

A partir de la curva de acumulación de especies, se esperaría encontrar una riqueza de aves mayor al aumentar ya sea el número de muestreos o el número de sitios (como mínimo 45 muestreos en el tiempo), sin embargo esta proyección estadística puede cambiar dependiendo de las variables biofísicas de los parques (la infraestructura, la vegetación, los estratos vegetales, el \% de áreas verdes y áreas grises, así como otras variables no contempladas incluyendo la intervención del ser humano), ya que estos son factores que pueden permitir o restringir esa proyección de especies esperadas. Se puede deducir que hacen falta más muestreos para llegar a la asíntota que señala el número extrapolado de especies para la zona de muestreo.

Los datos de abundancia por encima del promedio pueden deberse a la predominancia de Columba livia como especie más abundante, debido a varios factores: 1) Columba livia es una especie exótica abundante y distribuida en todo el mundo. 2) La facilidad de adaptarse al entorno humano. 3) Alimentación antropogénica (en el bulevar del correo no es evidente, como si lo es en el Parque Morazán y Parque Central). 4) Superioridad porcentual de área con obra gris a nivel de superficie (pavimentos) en estos sitios y la adaptabilidad de esta especie a estos entornos. El Parque Morazán presenta un 52.85\% de superficies con obra gris, el Parque Central 91,58\% y Bulevar del Correo $81,75 \%$. 5) Presencia de infraestructura (interna o externa) que brinda sitios de refugio y anidación a esta especie. 7) Baja presencia de depredadores naturales (no observados durante la investigación) (Fallas, 2016).

Con respecto a la abundancia, no se muestran evidencias que indiquen que la relación área/abundancia. Se evidencia una mayor abundancia de aves en parques donde predominan las áreas de obra gris. Además, la infraestructura también determina la riqueza biológica, en sitios con mayor presencia de infraestructuras o edificaciones (internas o externas al parque) favorece la abundancia de aves colonizadoras ya que las fachadas que rodean estos parques son construcciones con espacios utilizados como sitios de anidación y como dormitorios por Columba livia, mientras que el área de parque representa los sitios de alimentación para esta especie.

Según el principio de área y número de especies de la teoría insular de MacArthur y Wilson (1963), el tamaño del área por lo general permite la existencia de mayor o menor cantidad de hábitats disponibles, los cuales a su vez determinan el número de especies encontradas. A este principio se le puede bajar la escala y extrapolarlo a los entornos urbanos, donde el tamaño de los parques urbanos es uno de los condicionantes que permite la existencia de mayor o menor cantidad de hábitats disponibles, los cuales a su vez determinan el número de especies encontradas, generando un comportamiento similar al que puede suceder en sistemas insulares. Sin embargo, Almazán y Hinterholzer (2010) concuerdan con Blair (2001) y Crooks et al. (2004) los cuales afirman que la 
riqueza de aves en un lugar disminuye más por estar rodeado de áreas densamente edificadas que por tener una superficie pequeña, resultando en comunidades más homogéneas. Rojas (2014) concuerda con Lancaster y Rees (1979) quienes mencionan que la diversidad de aves se relaciona inversamente con el incremento de diversidad de hábitat, aportados por elementos de las construcciones humanas y mantienen que el alimento provisto por el hombre tuvo mayor efecto en la densidad y la distribución de las aves, para este estudio Columba livia.

Al relacionar riqueza y abundancia de aves, es evidente que la diferencia numérica correspondiente se ve condicionada básicamente por tres variables: el área del parque, la complejidad de la estructura vegetal y la cercanía a fragmentos de vegetación, así lo mencionan Soulé et al, (1988) y Fernández-Juricic (2001), quienes establecieron que la riqueza de especies de aves en los parques urbanos puede verse afectada por el tamaño del parque, la edad del sitio la cual es habitualmente relacionada positivamente con la complejidad en la estructura de vegetación del parque y por el aislamiento respecto del hábitat fuente. Es importante mencionar que parques con baja riqueza alteran los datos de la relación riqueza-abundancia. Estos parques presentan al menos una de las variables antes mencionadas (tamaño del parque (pequeño) o una complejidad estructural vegetal pobre, así como aislamiento con un hábitat fuente). Siendo estas las posibles causas de la alteración de los datos promedios en comparación con los restantes parque en estudio.

A partir el índice de similitud de Morisita se obtuvo dos grandes grupos (el primero subdividido en dos subgrupos). Un primer grupo (similitud aproximada al $90 \%$ ) con una riqueza inferior donde la riqueza sobresale Columba livia, Quiscalus mexicanus, Thraupis episcopus y tres especies migratorias. Estos parques presentan características favorables para la presencia especies frugívoras e insectívoras migratorias y árboles importantes como fuente de alimento directa e indirecta para este grupo de aves, como por ejemplo Cojoba arborea, Cecropia sp. La similitud en el primer subgrupo se genera debido a las marcadas diferencias en bajos porcentajes de área verde, alto porcentaje de áreas pavimentadas y bajos porcentajes tanto de riqueza como abundancia. La similitud en el segundo subgrupo se genera debido a las marcadas diferencias en bajos porcentajes de área verde, alto porcentaje de áreas pavimentadas (característica que comparten ambos subgrupos en el primer grupo), así como un alto porcentaje de abundancia y una riqueza intermedia según promedio de todos los parques estudiados. Un segundo grupo (similitud del 45\%) donde existe mayor diversidad de especies, presentan áreas con superioridad porcentual de espacios verdes o con presencia equitativa de áreas verdes y áreas con obra gris (pavimentos). En estos sitios no se evidencia abundancia de Columba livia y las especies observados corresponden mayormente a especies migratorias.

Se concluye que los 15 parques urbanos en estudio son sitios utilizados por la avifauna residente y migratoria como zonas de paso o estancia durante los procesos de migración, así como hábitat permanente para otras especies.

\section{AGRADECIMIENTOS}

Agradezco a Katya Castro Del Valle, Jonathan Arias, Adrián Damaceno ( $\nmid$ y Otto Monge.

\section{REFERENCIAS}

Almazán, C., \& Hinterholzar, A. (2010). Dinamica temporal de la avifauna en un parque urbano de la ciudad de Puebla, México. Huitzil, XI(1). Recuperado de http://www.scielo. org.mx/scielo.php?pid=S1870-74592010000100007\&script=sci_arttext

Arias, J. (7 de Octubre de 2009). Parques Urbanos, Municipalidad de San José. (A. A. Solano, Interviewer) San José, Costa Rica.

Artavia, R., \& Valle, D. (2013). Diagnóstico preliminar de avifauna para instaurar el Corredor Biológico Interurbano Río Torres en el cantón San José. Ambientico, 232-233, 56-63.

Barrantes, F. (1964). Reseña geográfica del cantón central de san José. San José, Costa Rica: Instituto Geográfico.

CHAO, A. (1987). Estimating the population size for capturerecapture data with unequal catchability. Biometrics, 43, 783-791.

Chazdon, R. L., Colwell, R. K., Denslow, J. S., \& Guariguata, M. R. (1998). Statistical methods for estimating species richness of woody regeneration in primary and secondary rain forests of NE Costa Rica. En Dallmeier F. \& J. A. Comiskey (eds.). Forest biodiversity research, monitoring and modelling: conceptual background and old world case studies ( pp. 285-309). Paris: Parthenon Publishing.

Colwell, R. K. (2013). EstimateS: Statistical estimation of species richness and shared species from samples. Version 9. Recuperado de http://purl.oclc.org/estimates.

De la Herra, I., Unanue, A., \& Aguirre, I. (2009). Efectos del área, edad y cobertura de la vegetación sobre la riqueza de especies de aves reproductoras en los parques urbanos de Vitoria-Gasteiz. Munibe Ciencias Naturales, 57, 195-206. 
Espinoza, C., Quan, C., Pacheco, C., Rizo, F., Francia, G., ... \& Suazo, J. (2002). Hábitat disponible para aves y saurios en Heredia urbano. Ambientico, 103, 14-16.

Fallas, A. (2016). Uso de parques urbanos por aves residentes y migratorias en la ciudad de San José, Costa Rica (Tesis de Licenciatura). Universidad Estatal a Distancia. San José, Costa Rica.

Fernández-Juricic, E., \& Jokimaki, J. (2001). A habitat island approach to conserving birds in urban landscapes: case studies from southerm and northern Europe. Biodiversity and Conservation, 10, 2023-2043.

González, M. (2009). Avifauna Urbana en América Latina: Estudios de Caso. Gestión Ambiental, 17, 55-68.

Gotelli, N. J., \& Colwell, R. K. (2010). Estimating species richness. En: Magurran, A. E. \& B. J. McGill (eds.). Biological diversity: frontiers in measurement and assessment (pp. 39-54). Oxford: Oxford University Press.

Hammer, O., Harper, D., \& Ryan, P. D. (2001). Past: Paleontological Statistics Software Package for Education and Data Analysis. Palaeontologia Electronica, 4(1), art. 4: Recuperado de http://palaeo-electronica.org/2001_1/ past/issue1_01.htm

Herzog, S. K., Kessler, M., \& Cahill, T. M. (2002). Estimating species richness of tropical bird communities from rapid assessment data. The Auk, 119,749-769.

Hortal, J., Borges, P. A. V., \& Gaspar, C. (2006). Evaluating the performance of species richness estimators: sensitivity to sample grain size. Journal of Animal Ecology, 75,274-287.

Leveau, L., \& Leveau, C. (2004). Comunidaddes de aves en un gradiente urbano de la ciudad de Mar de Plata, argentina. Hornero, 19(1), 13-21.

MacArthur, R. H., \&Wilson, E. O. (1963). An equilibrium theory of insular zoogeography. Evolution, 17(4), 373-387.

Murillo, M., \& León-Castellá, A. (2007). Costa Rica y sus provincias. Recuperado de http://www.cientec.or.cr/provincias/provincias.html

Obando-Calderón, G. J.-C. (Noviembre de 2014). Lista oficial de las aves de Costa Rica 2013-2014. Zeledonia, 17, 2.
Ralph, C., Geupel, G., Pyle, P., Martin, T., DeSante, D., \& Milá, B. (1996). Manual de métodos de campo para el monitoreo de aves terrestres. Albany, California, EEUU: Forest Service. Departament of Agriculture.

Ramírez, O., Amador, M., Camacho, L., Carranza, I., Chaves, E., ...\& Moya, A. (2008). Conocimiento popular de la paloma de Castilla (Columba livia) en el parque central de Alajuela. Zeledonia, 12 (1), 14-20.

Rojas, M. (2014). Diversidad y uso de hábitat de aves en diferentes gradientes urbanos en la ciudad de Guauaquil, Ecuador. Recuperado de http://repositorio.ug.edu.ec/bitstream/ redug/7137/1/TESIS\%20\%20MARIA\%20LORENA\%20 ROJAS.pdf

Sokal, R. R., \& Rohlf, F. J. (1995). Biometry. $3^{\text {rd }}$ edn. New York: W. H. Freeman.

Stiles, F. G., \& Skutch, F. A. (2003). Guía de Aves de Costar Rica. Santo Domingo, Heredia, Costa Rica: Instituto Nacional de Biodiversidad.

Ugland K. I., Gray, J. S., \& Ellingsen, K. E. (2003). The species-accumulation curve and estimation of species richness. Journal of Animal Ecology, 72, 888-897.

Urquiza, A., \& Mella, J. (2002). Riqueza y diversidad de aves en parques de Santiago durante el periodo estival. Boletín Chileno de Ornitología, 9, 12-21.

Walther, B. A., \& Morand, S. (1998). Comparative performance of species richness estimation methods. Parasitology, 116, 395-405.

Zavala, G., \& Gismondi, P. (2003). Variación de la riqueza y diversidad de la ornitifauna en áreas verdes urbanas de las ciudades de la Paz y El Alto (Bolivia). Ecología en Bolivia, XXXVIII(1). Recuperado de http://www.scielo.org.bo/ scielo.php?pid=S1605-25282003000100006\&script=sci_arttext

Sección de Parques y áreas verdes, Municipalidad de San José. Parques y áreas verdes, distritos centrales. Levantamiento de campo: SIG-PLANARBU. 2009. San José, CR. Esc. 1:10.000. Color. 